\title{
Identification of Potential Biomarkers and Metabolic Profiling of Serum in Ovarian Cancer Patients Using UPLC/Q-TOF MS
}

\author{
Wanchao Yang ${ }^{\mathrm{a}}$ Tian $\mathrm{Mu}^{\mathrm{b}}$ Jing Jiang ${ }^{\mathrm{c}}$ Qintong Sun ${ }^{\mathrm{b}}$ Xuejia $\mathrm{Hou}^{\mathrm{b}}$ \\ Yu Sun $^{d}$ Lei Zhong ${ }^{d}$ Cheng Wang ${ }^{b}$ Changhao Sun ${ }^{e}$ \\ aDepartment of Anesthesiology, Second Affiliated Hospital of Harbin Medical University, Harbin, \\ ${ }^{b}$ Department of Environmental Hygiene, School of Public Health, Harbin Medical University, Harbin, \\ 'Department of Obstetrics and Gynecology, Second Affiliated Hospital of Harbin Medical University, \\ Harbin, dDepartment of General surgery, Second Affiliated Hospital of Harbin Medical University, \\ Harbin, eDepartment of Nutrition and Food Hygiene, School of Public Health, Harbin Medical \\ University, Harbin, China
}

\section{Key Words}

Ovarian cancer $•$ Metabolomics $•$ Biomarkers $•$ Metabolic profiling

\begin{abstract}
Background/Aims: Ovarian cancer (OC) is a malignant neoplasm of the female reproductive system with a high mortality rate. Identifying useful biomarkers and clarifying the molecular pathogenesis of $O C$ are critical for early diagnosis and treatment. The aim of the study was to identify candidate biomarkers and explore metabolic changes of OC. Methods: A two-stage design was used in our study, with a discovery cohort of OC cases $(n=30)$ and controls $(n$ $=30)$ and an independent cohort of cases $(n=17)$ and controls $(n=18)$ for validation. The serum metabolic profiling was investigated by ultra-performance liquid chromatography and quadrupole time-of-flight mass spectrometry with positive electrospray ionization. Results: A total of 18 metabolites closely related to OC were identified in the discovery stage, of which 12 were confirmed in the validation cohort. Metabolic pathways in OC related to these biomarkers included fatty acid $\beta$-oxidation, phospholipid metabolism, and bile acid metabolism, which are closely related to the proliferation, invasion, and metastasis of cancer cells. Multiple logistic regression analysis of these metabolites showed that 2-piperidinone and 1-heptadecanoylglycerophosphoethanolamine were potential biomarkers of OC, with high sensitivity (96.7\%), specificity (66.7\%), and area under the receiver operating characteristic curve value (0.894). Conclusion: These findings provide insight into the pathogenesis pathogenesis of $\mathrm{OC}$ and may be useful for clinical diagnosis and treatment.
\end{abstract}

Cheng Wang

and Changhao Sun
Department of Environmental Hygiene; Department of Nutrition and Food Hygiene, School of Public Health, Harbin Medical University, Harbin 150081 (China)

E-Mail wangchenghlj@163.com; changhaosun2002@163.com 


\section{Cellular Physiology Cell Physiol Biochem 2018;51:1134-1148

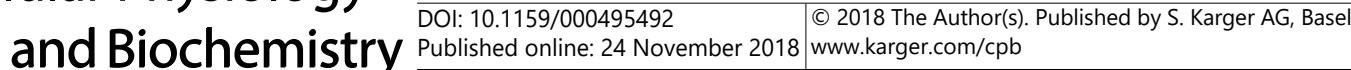 \\ Yang et al.: Metabolic Profiling of Serum in Ovarian Cancer}

\section{Introduction}

Ovarian cancer (OC) is a malignant neoplasm of the female reproductive system, and has a high global mortality rate. Worldwide, approximately 204, 000 new instances of OC are diagnosed each year, with 125,000 deaths due to $0 \mathrm{C}$ recorded annually [1]. Early-stage OC is difficult to diagnose due to the scarcity of symptoms, and its etiology is unclear [2]. As such, most patients are diagnosed at an advanced stage and have a low 5-year survival rate [3]. The assay for cancer antigen (CA)125, which is the U.S. Food and Drug Administrationapproved test for $\mathrm{OC}$ detection, has high false-positive and -negative rates. Other approaches for detecting $\mathrm{OC}$, for instance, based on carcinoembryonic antigen and human epididymis protein 4 expression, have similar problems [4]. It is therefore necessary to identify more specific biomarkers and catch molecular pathogenesis in order to facilitate the early detection of $\mathrm{OC}$ and evaluation of treatment.

Metabolomics is an unbiased analytical method for quantitative and qualitative analyses of metabolites in cells, tissue, or biofluids and their changes in response to pathophysiological stimuli [5-7]. As products and substrates of metabolism, metabolites play important roles in cellular functions including cell proliferation and apoptosis, which are correlated with the occurrence and development of cancer [8]. Some metabolomic studies of OC have recently been carried out using different techniques. An analysis of serum by liquid chromatography followed by mass spectrometry (LC-MS) identified 7-nor-5 $\beta$-cholestane-3, 7,12, 24, 25 pentol glucuronide as a potential biomarker for OC that is complementary to CA125 [9]. Another study using ${ }^{1} \mathrm{H}$-nuclear magnetic resonance spectroscopy showed that the levels of metabolites such as acetoacetate, acetone, and 3-hydroxybutyrate were elevated in OC patients [10]. Esterified fatty acid (EFA) (C16:0), EFA (C18:0), and free fatty acid (FFA) (C16:0) are also possible biomarkers that were identified by gas chromatographymass spectrometry (GC-MS) analysis, indicating that OC is associated with changes in FA metabolism [11]. Ultra-performance liquid chromatography (UPLC) coupled with MS has higher resolution, sensitivity, and a better metabolite detection range than other analytical techniques and has been widely used to investigate metabolic changes in cancer $[12,13]$.

In this study, we used UPLC quadrupole time-of-flight (Q-TOF) tandem MS (MS/MS) to identify potential serum biomarkers and explore metabolic changes in OC in a cohort of patients. The biomarkers were then validated in an independent OC case-control experiment.

\section{Materials and Methods}

\section{Study design and biospecimen collection}

The two-stage study design included a discovery cohort of OC cases $(n=30)$ and controls $(n=30)$, and an independent cohort of cases $(n=17)$ and controls $(n=18)$ for validation. OC was confirmed according to the International Federation of Gynecology and Obstetrics (FIGO) staging system [14], and control subjects were healthy, age-matched volunteers. Exclusion criteria were patients with metabolic, kidney, or liver disease or any other type of cancer. Serum collection and processing were carried out according to standard procedures. All subjects underwent an OC screening test prior to inclusion in the study. Before the screening test, venous blood samples were collected after overnight fasting for $12 \mathrm{~h}$ and centrifuged at $3000 \times g$ for 15 min to obtain serum, which was stored at $-80^{\circ} \mathrm{C}$ until analysis. After confirming a diagnosis of OC, the serum was assigned to the OC or control group. Written, informed consent was obtained from all study subjects and the study protocol was approved by the Ethics Committee of the Harbin Medical University (no. 2016005) and was in accordance with the principles outlined in the Declaration of Helsinki. 


\section{Cellular Physiology Cell Physiol Biochem 2018;51:1134-1148 \begin{tabular}{l|l|l} 
and Biochemistry 2018 The Author(s). Published by S. Karger AG, Basel & $\begin{array}{l}\text { DOI: 10.1159/000495492 } \\
\text { wublisher.karger.com/cpb }\end{array}$
\end{tabular}

Yang et al.: Metabolic Profiling of Serum in Ovarian Cancer

\section{Chemicals and reagents}

Chromatography-grade acetonitrile and methanol were from Honeywell Burdick \& Jackson (Muskegon, MI, USA). Analytical grade formic acid and leucine enkephalin were purchased from Beijing Reagent Company (Beijing, China), and Sigma-Aldrich (St Louis, MO, USA). Deionized water was purified using an ultra-clear system (SG Water Conditioning and Regeneration, Barsbüttel, Germany). Standards were obtained from commercial sources.

\section{Sample preparation}

Samples were prepared as previously described $[15,16]$. Before analysis, $350-\mu l$ serum samples were mixed with 1, $400 \mu \mathrm{l}$ methanol at $4^{\circ} \mathrm{C}$. The mixture was vortexed for $3 \mathrm{~min}$, allowed to stand for $10 \mathrm{~min}$, and centrifuged at $14,000 \times g$ for $10 \mathrm{~min}$. The supernatant was dried with nitrogen and dissolved in 350 $\mu \mathrm{l}$ acetonitrile/water $(4: 1, \mathrm{v} / \mathrm{v})$, vortexed for $3 \mathrm{~min}$, allowed to stand for $10 \mathrm{~min}$, then centrifuged at 14, $000 \times g$ for $10 \mathrm{~min}$. The supernatant was transferred to autosampler vials. To verify the reproducibility and reliability of the data, a pooled quality control (QC) sample was prepared by mixing equal volumes of serum sample from 10 healthy subjects and $10 \mathrm{OC}$ patients, and injecting the mixture at every 10 th sample throughout the run. A randomized crossover design was used to avoid order effects in statistical analysis. Samples of five healthy subjects and five OC patients in the two analyzed batches were alternately injected.

\section{UPLC-QTOF-MS/MS conditions}

UPLC-Q-TOF-MS/MS analysis was performed as previously described $[15,16]$ with an Acquity UPLC system coupled with a Q-TOF-MS/MS system in electrospray ionization (ESI) in positive mode (Waters, Milford, MA, USA). A 2- $\mu \mathrm{l}$ sample was injected into a BEH-C18 column (4.6 $\times 50 \mathrm{~mm}, 1.7 \mu \mathrm{m}$; Waters). The mobile phase consisted of solvent A ( $0.1 \%$ formic acid in water) and solvent B (acetonitrile), and the flow rate was $0.3 \mathrm{ml} / \mathrm{min}$. The initial composition of $\mathrm{B}$ was $2 \%$ and increased to $20 \%$ from $0-1.5 \mathrm{~min}$, then from 20\%-70\% from 1.5-6 min, 70\%-92\% from 6-9 min, 92\%-98\% from 9-15 min, and 98\%-98\% from 15$16.5 \mathrm{~min}$, followed by re-equilibration to the initial conditions in $4 \mathrm{~min}$. For MS analysis in positive ESI mode, the capillary voltage and sample cone voltage were set at $3000 \mathrm{~V}$ and $35 \mathrm{~V}$, respectively. The desolvation temperature was $300^{\circ} \mathrm{C}$ and the gas flow rate was $600 \mathrm{l} / \mathrm{h}$. The source temperature was set at $100^{\circ} \mathrm{C}$ and the cone gas flow was $50 \mathrm{l} / \mathrm{h}$. Centroid data were collected from $80-1000 \mathrm{~m} / \mathrm{z}$ at an acquisition rate of 0.4 with a 0.1-s interscan delay. To ensure accuracy and reproducibility, leucine enkephalin at $200 \mathrm{pg} / \mathrm{ml}$ was used as the lock-mass for positive ESI mode $([\mathrm{M}+\mathrm{H}]+=556.2771)$ via a lock spray interface.

\section{Data processing and biomarkers screening}

Data were processed as previously described $[15,16]$. The UPLC/Q-TOF MS data were converted in MarkerLynx Application Manager 4.1 SCN 714 (Waters) for analysis. The mass window was set at $0.02 \mathrm{Da}$, noise elimination level at 10.00 , retention time tolerance at $0.01 \mathrm{~min}$, and retention time window at $0.2 \mathrm{~min}$. Data were used up to $16.5 \mathrm{~min}$ after the start of the column-washing phase. The resultant three-dimensional matrix consisting of peak indices (retention time-m/z pairs), sample names (observations), and normalized ion intensities for each peak area was exported to EZINFO 2.0 (a component of MarkerLynx) to analyze grouping trends and outliers by the score plot and determine variable importance in projection (VIP) values by partial least squares discriminant analysis (PLS-DA). A principal components analysis (PCA) was used to obtain an overview of data quality assessment. Potential biomarkers were selected according to VIP value (> 1.5) [17]. The goodness-of-fit was quantified by $R^{2} Y$, while the predictive ability was determined as $Q^{2}$. Using SIMCA-P software (v.11.5; Umetrics AB, Umeå, Sweden), a-cross validation procedure and testing with 800 random permutations was performed to avoid the over-fitting of supervised PLS-DA models. To validate the most significant biomarkers and evaluate classification performance, multiple logistic regression analysis of top candidate metabolites was performed by stepwise selection. The predictive ability of potential metabolic biomarkers and area under the curve (AUC) were evaluated by receiver operating characteristics (ROC) analysis. Sensitivity and specificity were determined according to the maximum value of the Youden index.

The identification of empirical formulae for potential biomarkers was first performed according to accurate mass measurement and the relative intensities of isotopic peaks in high-resolution mass spectra. The MS/MS fragment ion-analysis procedure was facilitated by chemically intelligent peak-matching algorithms using the MassFragment application manager (MassLynx v.4.1, Waters). Briefly, MS/MS spectra of metabolites and exact mass were matched with the structural information of metabolites in the Human 


\section{Cellular Physiology Cell Physiol Biochem 2018:51:1134-1148

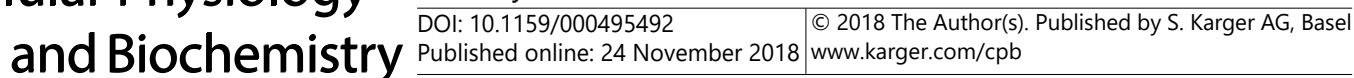 \\ Yang et al.: Metabolic Profiling of Serum in Ovarian Cancer}

Metabolite Database (HMDB) based on parameters such as deviation from calculated mass double bond equivalent and i-fit value (isotopic pattern of the selected ion). The candidate biomarkers were conformed with standard compounds based on retention time and tandem mass spectra. The pathways for biomarkers were interpreted using information from HMDB (http://www.hmdb.ca), Kyoto Encyclopedia of Genes and Genomes (http://www.genome.jp/kegg/) and references.

\section{Statistical analysis}

Statistical analysis was performed using SPSS v.13.01S (SPSS Inc., Chicago, IL, USA). Data are presented as the mean \pm standard deviation (SD). An independent samples $t$-test was used to analyze differences between groups. P values were two-tailed and a value $<0.05$ was considered as significant.

\section{Results}

\section{Clinical characteristics of study subjects}

In this study, we recruited a discovery cohort of $30 \mathrm{OC}$ patients and 30 healthy controls and a validation cohort of 17 OC patients and 18 healthy controls. There were no significant differences in age and weight between OC and control groups. OC patients had higher serum levels of CA125 than healthy individuals. OC was classified into four stages according to FIGO staging system (Table 1).

\section{Data quality assessment of metabolomics platform}

An overview of the quality of the analytical run was obtained by PCA of the sample and QC injections dataset. The results showed that the QC samples were tightly clustered in the score plots (Fig. 1, red crosses). In the discovery samples, the relative (R) SDs of retention time and peak intensity ranged from $0.07 \%$ to $0.63 \%$ and $4.23 \%$ to $6.56 \%$, respectively. In the validation samples, the RSDs of retention time and peak intensity ranged from $0.03 \%$ to $0.67 \%$ and $2.36 \%$ to $8.82 \%$, respectively (Table 2). These values were all lower than $10 \%$, implying that the results had excellent repeatability.

Table 1. Demographic and clinical characteristics of participants in the discovery and validation studies. ${ }^{\dagger}$ These patients were documented with "adenocarcinoma", without further subtype information.* Compared with OC patients, $p<0.05$

\begin{tabular}{|c|c|c|c|c|c|}
\hline \multirow{2}{*}{\multicolumn{2}{|c|}{ Category }} & \multicolumn{2}{|c|}{ Discovery cohort } & \multicolumn{2}{|c|}{ Validation cohort } \\
\hline & & $\mathrm{OC}$ & Control & $\mathrm{OC}$ & Control \\
\hline Number of subjects & & 30 & 30 & 17 & 18 \\
\hline Age (years) & & $54.7 \pm 11.54$ & $55.82 \pm 10.94$ & $52.63 \pm 11.81$ & $54.16 \pm 9.58$ \\
\hline Weight $(\mathrm{kg})$ & & $60.18 \pm 5.82$ & $62.33 \pm 7.44$ & $63.57 \pm 6.49$ & $64.07 \pm 4.30$ \\
\hline CA125(U/ml, median, $\mathrm{r}$ & & $721.18(56.4-4315.22)$ & $21.85(10.23-47.64) *$ & $662.25(23.47-4870.63)$ & $24.2(13.11-59.37)^{*}$ \\
\hline \multirow[t]{2}{*}{ Menopause (pre/post) } & Premenopause & 7 & 9 & 2 & 5 \\
\hline & Postmenopause & 23 & 21 & 15 & 13 \\
\hline \multicolumn{6}{|l|}{ Stage } \\
\hline & I & 2 & & 3 & \\
\hline & II & 1 & & 1 & \\
\hline & III & 25 & & 10 & \\
\hline & IV & 2 & & 3 & \\
\hline \multicolumn{6}{|l|}{ Histology type } \\
\hline & Serous & 24 & & 12 & \\
\hline & Mucoid & 2 & & 1 & \\
\hline & Endometrioid & 1 & & 3 & \\
\hline & Clear cell & 1 & & 1 & \\
\hline & Other ${ }^{\dagger}$ & 2 & & 0 & \\
\hline \multicolumn{6}{|l|}{ Histology differentiation } \\
\hline & Well differentiated & 5 & & 1 & \\
\hline & Moderately differentiated & 3 & & 2 & \\
\hline & Poorly differentiated & 22 & & 14 & \\
\hline
\end{tabular}




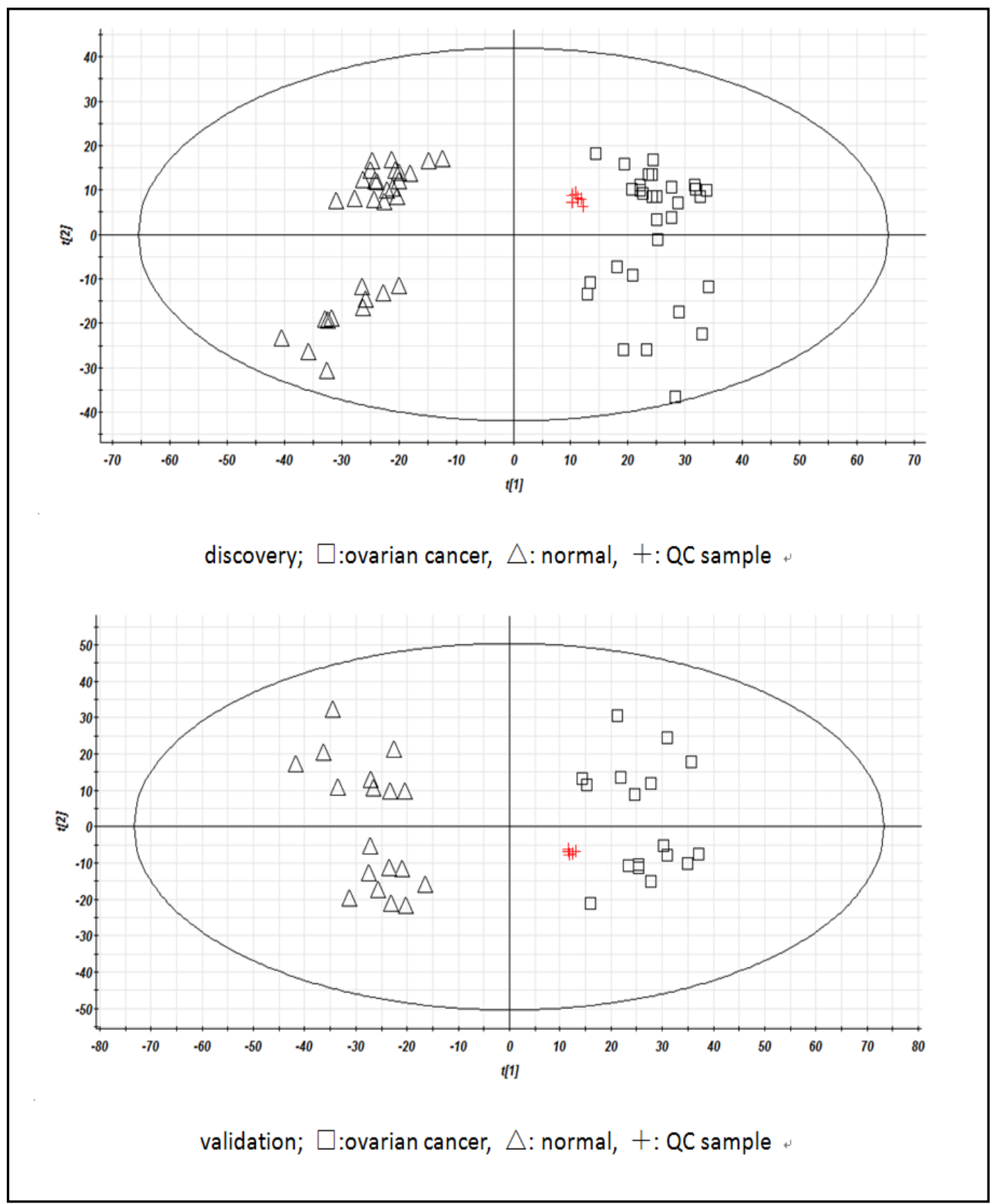

Fig. 1. PCA scores plot of ovarian cancer, control and quality control (QC) samples in the discovery and validation stages. $\mathrm{t}[1]=$ component $1 ; \mathrm{t}[2]=$ component 2 .

\section{Serum metabolite profiling}

Representative single UPLC-Q-TOF/MS base peak intensity chromatograms of an OC patient and a healthy control are shown in Fig. 2. A total of 6948 ions (peaks) were assessed in the discovery analysis. Both the unsupervised (PCA) and supervised (PLS-DA) methods were used to evaluate global metabolic alterations in healthy controls and OC patients, and corresponding VIP values were also estimated in the PLS-DA model. The PLS-DA score plots revealed that the $O C$ patients and healthy subjects could be separated into distinct clusters according to differences in serum metabolite composition, and that the parameters of the PLS-DA model-including the values of $\mathrm{R}^{2} \mathrm{Y}$ and $\mathrm{Q}^{2}$ ( 0.996 and 0.988 , respectively, in positive 
ESI mode) were all $>0.5$ (Fig. 3A), implying that the model was a good fit. In addition, the permutation test of the established PLS-DA model showed that all permuted $\mathrm{R}^{2}$ and $\mathrm{Q}^{2}$ values were lower than the original points to the right and that the $\mathrm{Q}^{2}$ regression line had a negative intercept (Fig. 3B), indicating the feasibility of the PLS-DA model.

\section{Identification of differential metabolites}

The VIP values (VIP > 1.5) obtained from the established PLS-DA modeland Pvalues $(\mathrm{P}<0.05)$ obtained from the $t$-test of metabolites in the two groups were used as a basis for screening candidate metabolites. A total of 18 metabolites were identified including 2-piperidinone, eicosadienoic acid, 7-ketodeoxycholic acid, varanic acid, 1-heptadecanoylglycerophosphoethanolamine, lysophosphatidylcholine (lysoPC) (P-16:0), lysoPC (15:0), lysophosphatidylethanolamine (lysoPE) (0:0/20:0), 2-hexaprenyl-3-methyl-6-methoxy-1, 4-benzoquinone, lysoPC (22:6), diacylglycerol (DG) (14:0/20:0/0:0), PE (18:4/P-16:0), dimethylphosphatidylethanolamine (PE-NMe2) (18:1/18:1), PC (14:0/22:4), PC (14:1/22:2), 3-0-sulfogalactosylceramide (d18:1/18:1), galabiosylceramide (d18:1/18:1), and lactosylceramide (d18:1/22:0) (Table 3). More detailed information on each metabolite is provided in Table 4. Five of the 18 metabolites including PC (14:1/22:2), eicosadienoic acid, varanic acid, lysoPC (15:0), and lysoPE (0:0/20:0) were
Table 2. Repeatability of experimental method in discovery and validation studies. $\mathrm{RT}=$ retention time; $\mathrm{RSD}=$ relative standard deviation

\begin{tabular}{lcccc}
\hline \multicolumn{5}{c}{ Repeatability } \\
m/z & $\begin{array}{c}\text { RT, min } \\
\text { Mean RSD (\%) }\end{array}$ & Mean & RSD (\%) \\
\hline $\begin{array}{l}\text { Discovery } \\
100.12\end{array}$ & 2.11 & 0.24 & 664.45 & 5.69 \\
158.19 & 5.21 & 0.10 & 15.65 & 5.99 \\
226.98 & 0.65 & 0.63 & 55.71 & 4.23 \\
525.69 & 8.49 & 0.05 & 1393.32 & 4.36 \\
896.34 & 12.61 & 0.07 & 1.14 & 6.56 \\
Validation & & & & \\
80.85 & 0.67 & 0.67 & 16.91 & 5.64 \\
265.05 & 6.83 & 0.08 & 344.22 & 7.15 \\
545.12 & 2.73 & 0.16 & 69.92 & 2.36 \\
690.35 & 9.93 & 0.05 & 2.28 & 4.45 \\
899.49 & 14.36 & 0.03 & 0.92 & 8.82 \\
\hline \multicolumn{7}{c}{} \\
\hline
\end{tabular}

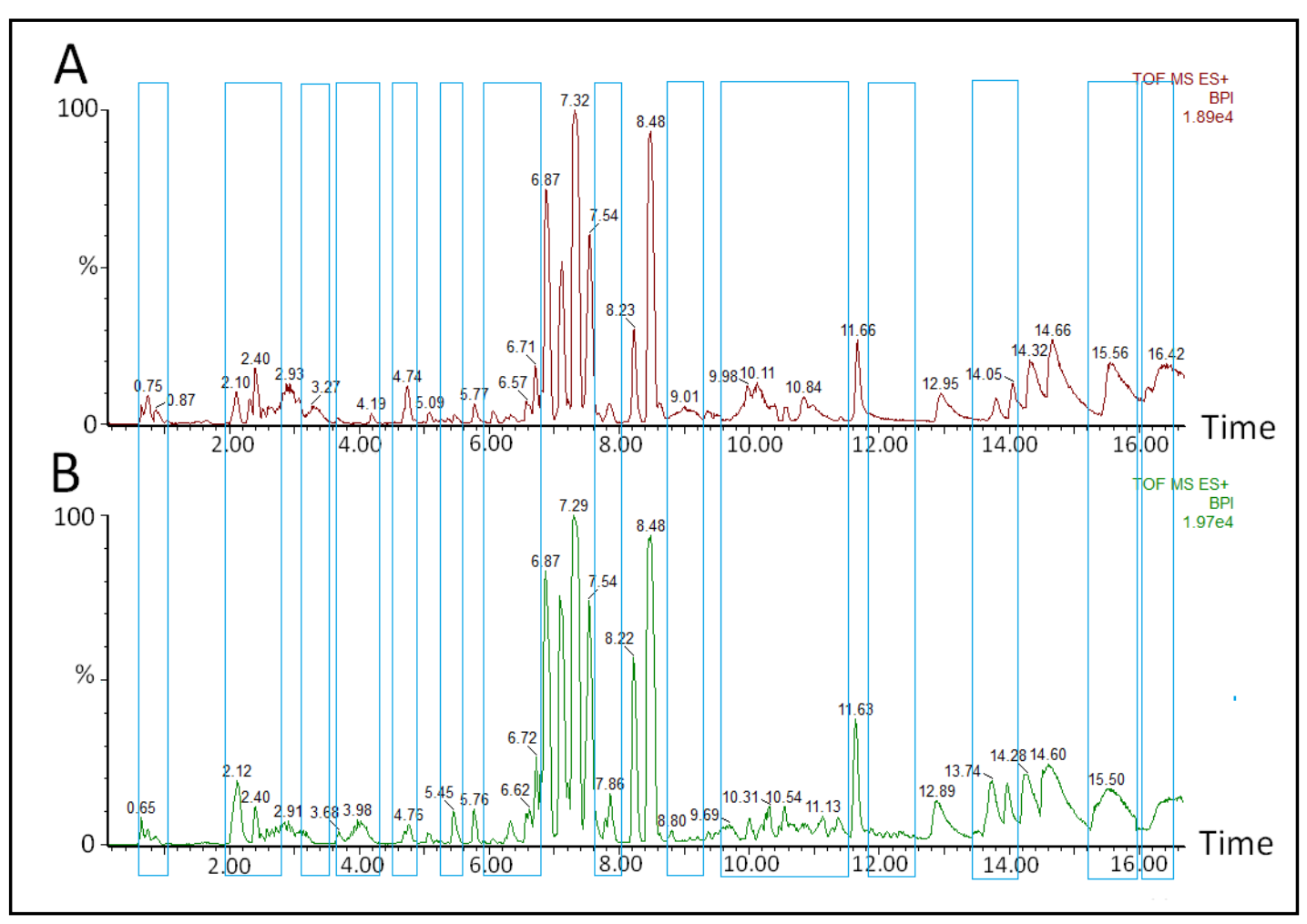

Fig. 2. Typical serum intensity (BPI) chromatograms in ovarian cancer and control groups. (A) ovarian cancer versus (B) control sample. The $\mathrm{Y}$ axis and $\mathrm{X}$ axis shows the relative abundance $(\%)$ and the retention time in minutes, respectively. Blue box marked the differences between the two chromatograms. 


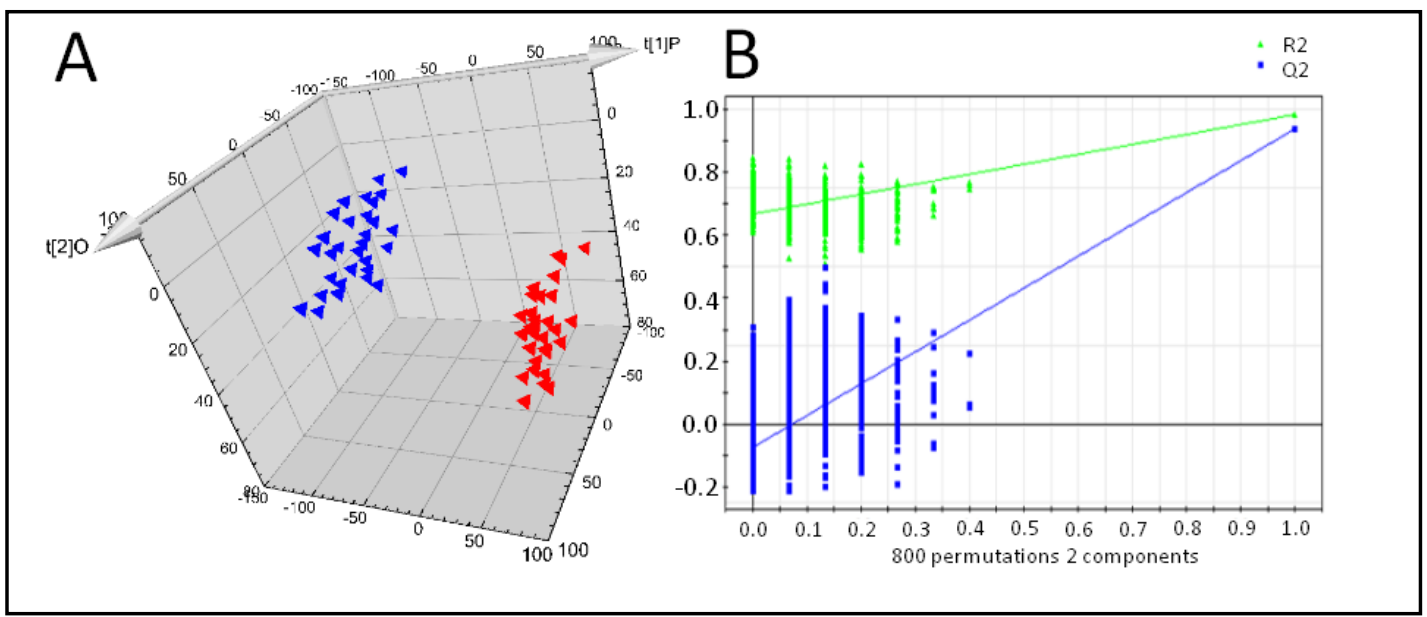

Fig. 3. A: 3-dimensional score plot with PLS-DA in patients with ovarian cancer and healthy controls. $R^{2} Y=$ $0.996, Q^{2}=0.988$. Controls are indicated in red, and patients with ovarian cancer in blue. $t[1]=$ first principal component; $\mathrm{t}[2]$ = second principal component; $\mathrm{B}$ : The result of permutation test on the PLS-DA model in positive ESI mode. The $\mathrm{Y}$ axis shows $\mathrm{R}^{2} \mathrm{Y}$ and $\mathrm{Q}^{2}$, and the $\mathrm{X}$ axis shows the correlation of observed and permuted data. The R2Y and Q2 value represent the goodness of fit and the predictability of the model. All $\mathrm{R}^{2} \mathrm{Y}$ and $\mathrm{Q}^{2}$ values on the permuted data set to the left were lower than original points to the right, and the regression line of $\mathrm{Q}^{2}$ has a negative value of intercept on the $\mathrm{Y}$ axis. The results showed that the model was valid.

Table 3. Metabolites identified as differentially expressed in the positive ESI mode in the discovery cohort. $\mathrm{RT}$ = retention time; VIP = variable importance in the projection. * Molecular weight of metabolites from UPLC-MS/MS. † Calculated using the molecular weight calculator in Mass Lynx version 4.1. $¥$ (Calculated mass - measured mass $) \div$ measured mass $\times 1000000$. $\S$ Ratio of mean relative amount between the ovarian cancer and control groups

\begin{tabular}{|c|c|c|c|c|c|c|c|}
\hline $\mathrm{RT}$, min & $\begin{array}{l}\text { Measured } \\
\text { mass, Da* }\end{array}$ & $\begin{array}{c}\text { Calculated } \\
\text { mass, Da } \dagger\end{array}$ & $\begin{array}{c}\text { Mass error, } \\
\text { ppm } \neq\end{array}$ & $\begin{array}{c}\text { Elemental } \\
\text { composition }\end{array}$ & $\begin{array}{c}\text { Postulated } \\
\text { identity }\end{array}$ & VIP & $\begin{array}{c}\text { Fold } \\
\text { change§ }\end{array}$ \\
\hline 2.17 & 100.0759 & 100.0762 & 2.9977 & $\mathrm{C}_{5} \mathrm{H}_{9} \mathrm{NO}$ & 2-Piperidinone & 19.12 & 0.49 \\
\hline 9.94 & 309.2819 & 309.2794 & -8.0832 & $\mathrm{C}_{20} \mathrm{H}_{36} \mathrm{O}_{2}$ & Eicosadienoic acid & 11.70 & 1.46 \\
\hline 5.86 & 407.272 & 407.2797 & 18.9063 & $\mathrm{C}_{24} \mathrm{H}_{38} \mathrm{O}_{5}$ & 7-Ketodeoxycholic acid & 5.15 & 1.37 \\
\hline 10.4 & 437.3214 & 437.3267 & 12.1192 & $\mathrm{C}_{26} \mathrm{H}_{44} \mathrm{O}_{5}$ & Varanic acid & 4.56 & 1.80 \\
\hline 6.36 & 468.3077 & 468.3090 & 2.7760 & $\mathrm{C}_{22} \mathrm{H}_{46} \mathrm{NO}_{7} \mathrm{P}$ & 1-Heptadecanoylglycerophosphoethanolamine & 7.36 & 2.67 \\
\hline 7.64 & 480.349 & 480.3454 & -7.4946 & $\mathrm{C}_{24} \mathrm{H}_{50} \mathrm{NO}_{6} \mathrm{P}$ & LysoPC(P-16:0) & 5.55 & 0.72 \\
\hline 6.89 & 482.3234 & 482.3247 & 2.6953 & $\mathrm{C}_{23} \mathrm{H}_{48} \mathrm{NO}_{7} \mathrm{P}$ & LysoPC(15:0) & 5.31 & 0.72 \\
\hline 7.91 & 510.3559 & 510.3560 & 0.1959 & $\mathrm{C}_{25} \mathrm{H}_{52} \mathrm{NO}_{7} \mathrm{P}$ & LysoPE(0:0/20:0) & 7.51 & 0.69 \\
\hline 10.02 & 561.4239 & 561.4308 & 12.2902 & $\mathrm{C}_{38} \mathrm{H}_{56} \mathrm{O}_{3}$ & 2-Hexaprenyl-3-methyl-6-methoxy-1,4 benzoquinone & 5.48 & 0.50 \\
\hline 6.83 & 568.3378 & 568.3403 & 4.3988 & $\mathrm{C}_{30} \mathrm{H}_{50} \mathrm{NO}_{7} \mathrm{P}$ & LysoPC(22:6) & 5.45 & 0.18 \\
\hline 9.88 & 597.5415 & 597.5458 & 7.1962 & $\mathrm{C}_{37} \mathrm{H}_{72} \mathrm{O}_{5}$ & DG(14:0/20:0/0:0) & 5.16 & 1.76 \\
\hline 10.34 & 696.4882 & 696.4968 & 12.3477 & $\mathrm{C}_{39} \mathrm{H}_{70} \mathrm{NO}_{7} \mathrm{P}$ & $\mathrm{PE}(18: 4 / \mathrm{P}-16: 0)$ & 7.82 & 1.54 \\
\hline 8.27 & 772.5853 & 772.5856 & 0.3883 & $\mathrm{C}_{43} \mathrm{H}_{82} \mathrm{NO}_{8} \mathrm{P}$ & PE-NMe2(18:1/18:1) & 10.68 & 1.43 \\
\hline 14.59 & 782.5724 & 782.5700 & -3.0668 & $\mathrm{C}_{44} \mathrm{H}_{80} \mathrm{NO}_{8} \mathrm{P}$ & $\mathrm{PC}(14: 0 / 22: 4)$ & 7.71 & 0.79 \\
\hline 10.22 & 784.5869 & 784.5856 & -1.6569 & $\mathrm{C}_{44} \mathrm{H}_{82} \mathrm{NO}_{8} \mathrm{P}$ & $\operatorname{PC}(14: 1 / 22: 2)$ & 10.51 & 2.02 \\
\hline 13.95 & 806.5756 & 806.5630 & -15.6216 & $\mathrm{C}_{42} \mathrm{H}_{79} \mathrm{NO}_{13}$ & 3-0-Sulfogalactosylceramide (d18:1/18:1) & 6.22 & 0.27 \\
\hline 9.04 & 888.6672 & 888.6412 & -29.2573 & $\mathrm{C}_{48} \mathrm{H}_{89} \mathrm{NO}_{13}$ & Galabiosylceramide (d18:1/18:1) & 8.71 & 2.11 \\
\hline 9.4 & 946.7147 & 946.7195 & 5.0702 & $\mathrm{C}_{52} \mathrm{H}_{99} \mathrm{NO}_{13}$ & Lactosylceramide (d18:1/22:0) & 7.95 & 1.82 \\
\hline
\end{tabular}


Table 4. Relative contents of 18 differential metabolites between patients with ovarian cancer and healthy controls in discovery and validation cohort.

\begin{tabular}{|c|c|c|c|c|c|c|c|c|c|c|c|c|c|c|c|c|c|c|}
\hline \multirow{3}{*}{ Metabolites } & \multicolumn{9}{|c|}{ Discovery cohort } & \multicolumn{9}{|c|}{ Validation cohort } \\
\hline & \multicolumn{4}{|c|}{ Ovarian cancer $(n=30)$} & \multicolumn{4}{|c|}{ Control $(\mathrm{n}=30)$} & & \multirow[b]{2}{*}{ mean } & \multicolumn{4}{|c|}{ Ovarian cancer $(n=17)$} & \multicolumn{4}{|c|}{ Control $(n=18)$} \\
\hline & mean & $\min$ & $\max$ & med & mean & $\min$ & $\max$ & med & & & $\min$ & $\max$ & med & mean & $\min$ & $\max$ & med & $r$ \\
\hline 2-Piperidinone & 93.40 & 14.98 & 234.53 & 93.72 & 171.41 & 120.19 & 296.29 & 185.93 & $3<0.001$ & 1 69.13 & 30.52 & 155.36 & 58.92 & 174.07 & 762.36 & 313.52 & 2183.52 & $2<0.001$ \\
\hline icosadic & 364.13 & 123.45 & 578.36 & 374.44 & 249.57 & 7108.40 & 411.76 & 247.68 & $3<0.001$ & 1336.43 & 115.65 & 596.66 & 352.33 & 189.24 & 451.65 & 337.56 & 6202.09 & $9<0.001$ \\
\hline -Ketodeox & 78.37 & 19.37 & 167.97 & 67.99 & 57.24 & 11.51 & 142.04 & 45.73 & 0.028 & 73.32 & 21.33 & 152.36 & 68.16 & 43.99 & 8.41 & 97.52 & 39.55 & 0.007 \\
\hline Varanic acid & 45.69 & 2.74 & 141.08 & 36.62 & 25.41 & 9.29 & 76.15 & 21.87 & 0.005 & 43.57 & 1.86 & 101.52 & 37.85 & 19.51 & 3.52 & 53.59 & 17.09 & 0.009 \\
\hline rophosphoethanolamine & 48.72 & 14.34 & 119.42 & 45.74 & 18.22 & 2.81 & 37.61 & 13.50 & $<0.001$ & 42.12 & 12.52 & 71.52 & 49.39 & 13.64 & 3.75 & 35.36 & 12.32 & $<0.001$ \\
\hline LysoP & 93.47 & 42.43 & 148.94 & 90.41 & 130.09 & 9 54.02 & 213.12 & 124.71 & $1<0.001$ & 125.75 & 59.36 & 235.31 & 119.34 & 130.15 & 81.32 & 168.65 & 5125.36 & $6 \quad 0.739$ \\
\hline Lyso & 52.24 & 11.41 & 103.68 & 50.20 & 72.87 & 12.78 & 113.63 & 79.83 & 0.001 & 51.51 & 20.42 & 90.02 & 55.55 & 81.52 & 52.22 & 131.25 & 580.52 & $<0.001$ \\
\hline yso & 138.41 & 52.74 & 275.71 & 135.55 & 199.30 & 62.38 & 337.48 & 207.61 & $1<0.001$ & 155.21 & $1 \quad 69.70$ & 290.24 & 142.21 & 215.27 & 7102.32 & 2356.22 & 2220.12 & 20.014 \\
\hline $2-\mathrm{He}$ & 44.69 & 10.24 & 142.08 & 43.78 & 90.06 & 1.78 & 264.14 & 64.96 & $<0.001$ & 138.18 & 11.25 & 86.58 & 41.54 & 66.00 & 23.36 & 132.35 & $5 \quad 61.55$ & .008 \\
\hline so & 44.34 & 2.57 & 198.14 & 37.92 & 244.06 & 6146.76 & 467.01 & 235.20 & $<<0.001$ & 1223.92 & 2135.32 & 321.25 & 233.35 & 263.91 & 1149.10 & 0477.76 & 6248.37 & 148 \\
\hline & 52.54 & 1.82 & 117.09 & 47.87 & 29.88 & 12.34 & 70.59 & 24.71 & $<0.001$ & 156.07 & 1.03 & 147.14 & 44.52 & 26.16 & 5.46 & 57.13 & 25.38 & 0.025 \\
\hline & 12.02 & 17.63 & 207.99 & 109.35 & 72.96 & 31.42 & 125.73 & 70.77 & $<0.001$ & 102.85 & $\begin{array}{lll}5 & 11.37\end{array}$ & 201.25 & 108.34 & 78.57 & 28.36 & 199.65 & 566.35 & 0.226 \\
\hline & 01.30 & 59.84 & 449.47 & 193.55 & 140.54 & $\begin{array}{l}429.89 \\
\end{array}$ & 283.25 & 122.18 & $\begin{array}{ll}3 & 0.003\end{array}$ & 226.23 & $\begin{array}{ll}3 & 54.56\end{array}$ & 456.68 & 239.95 & 187.57 & 7118.99 & 9379.65 & 5145.65 & $\begin{array}{ll}5 & 0.246\end{array}$ \\
\hline & 492.01 & 258.56 & 965.00 & 480.19 & 620.65 & 5349.33 & 1073.01 & 1629.36 & 0.007 & 546.30 & o 333.34 & 967.59 & 539.42 & 629.78 & 3 321.25 & 5998.97 & 7647.54 & $4 \quad 0.220$ \\
\hline & 189.24 & 28.86 & 372.86 & 176.47 & 93.76 & 18.43 & 213.45 & 85.09 & $<0.001$ & 174.39 & 925.37 & 356.21 & 163.37 & 105.68 & 312.36 & 230.66 & 6125.49 & $\begin{array}{l}9 \\
9\end{array}$ \\
\hline $3-c$ & 91.25 & 29.28 & 162.29 & 93.55 & 338.02 & 2141.85 & 553.16 & 347.36 & $6<0.001$ & 194.54 & 25.64 & 171.15 & 100.12 & 88.77 & 36.32 & 158.59 & 988.52 & 0.652 \\
\hline & 179.62 & 22.21 & 394.62 & 153.27 & 85.00 & 9.02 & 179.53 & 88.27 & $<0.001$ & 170.92 & 219.65 & 356.46 & 145.56 & 67.04 & 15.33 & 175.57 & 754.21 & 0.001 \\
\hline & 120.22 & 8.42 & & 120.22 & 5.90 & 5.43 & 138.83 & 62.97 & $<0.001$ & 157.29 & $\begin{array}{ll}9 & 65.52 \\
\end{array}$ & 3.65 & 147.55 & 68.94 & 2.53 & 142 & 88.5 & \\
\hline
\end{tabular}

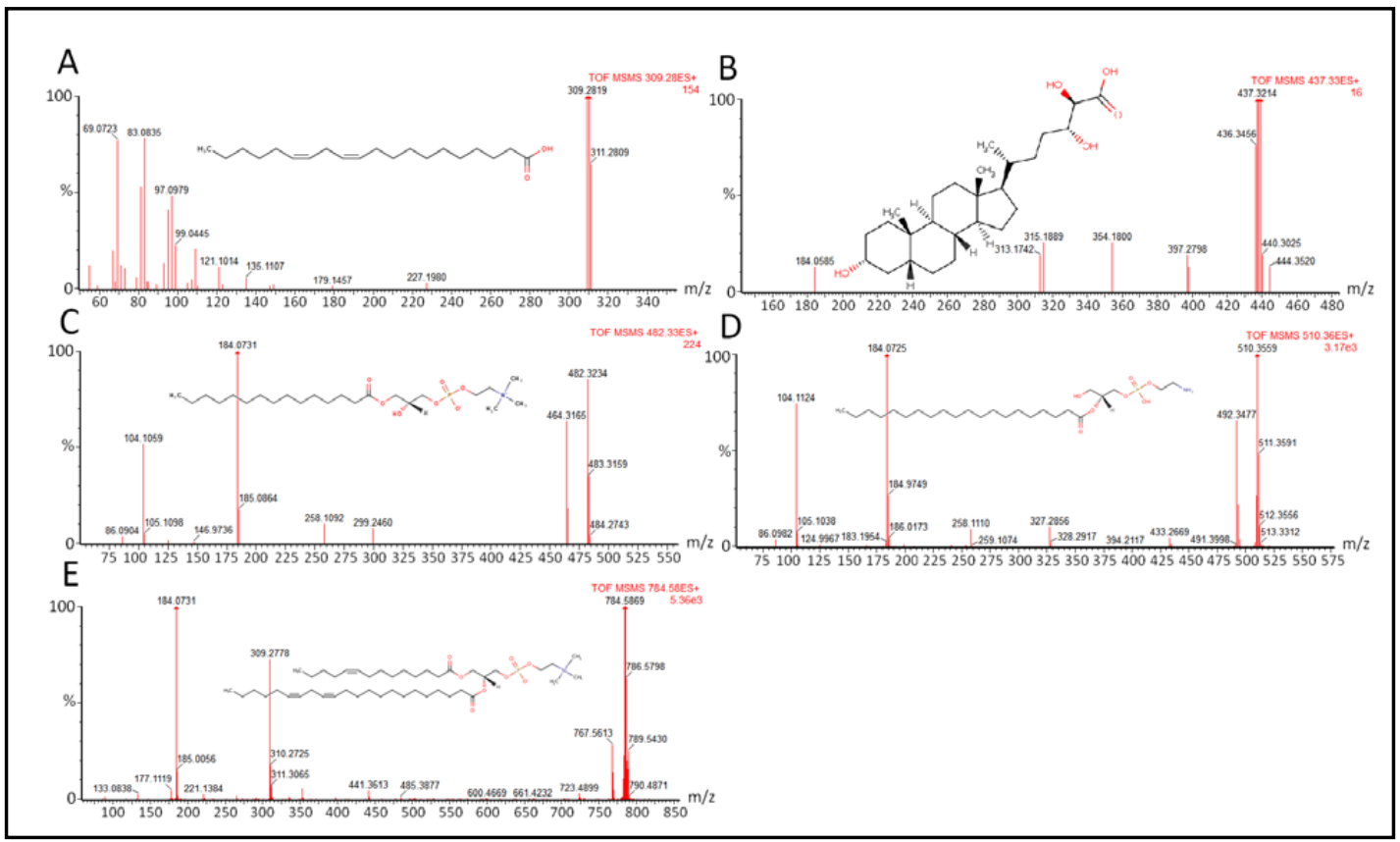

Fig. 4. Chemical structure and mass fragment information of 5 principal metabolites. (A) PC(14:1/22:2), (B) Eicosadienoic acid, (C) Varanic acid, (D) LysoPC(15:0), and (E) LysoPE(0:0/20:0).

identified using standard compounds. Chemical structures and mass fragment information on the principal metabolites are shown in Fig. 4. Other metabolites were confirmed by MS/ MS fragment ion-analysis and using a library database, since no authentic standards were commercially available (Fig. 5 and 6).

\section{Validation study of top metabolites}

An independent case-control experiment was performed to validate the 18 metabolites obtained in the discovery analysis. As in the latter, the experiment showed good reproducibility (Table 2). Differences in the abundance of the 18 metabolites between OC patients and controls in the validation group were evaluated (Table 4); the results showed that 12 of the metabolites differed significantly, including 2-piperidinone, eicosadienoic acid, 7-ketodeoxycholic acid, varanic acid, 1-heptadecanoylglycerophosphoethanolamine, 2-hexaprenyl-3-methyl6-methoxy-1, 4benzoquinone, lysoPC (15:0), lysoPE (0:0/20:0), DG (14:0/20:0/0:0), PC (14:1/22:2), galabiosylceramide (d18:1/18:1), and lactosylceramide (d18:1/22:0). Six metabolites were not validated. The AUC for the 12 metabolites showed good predictability 


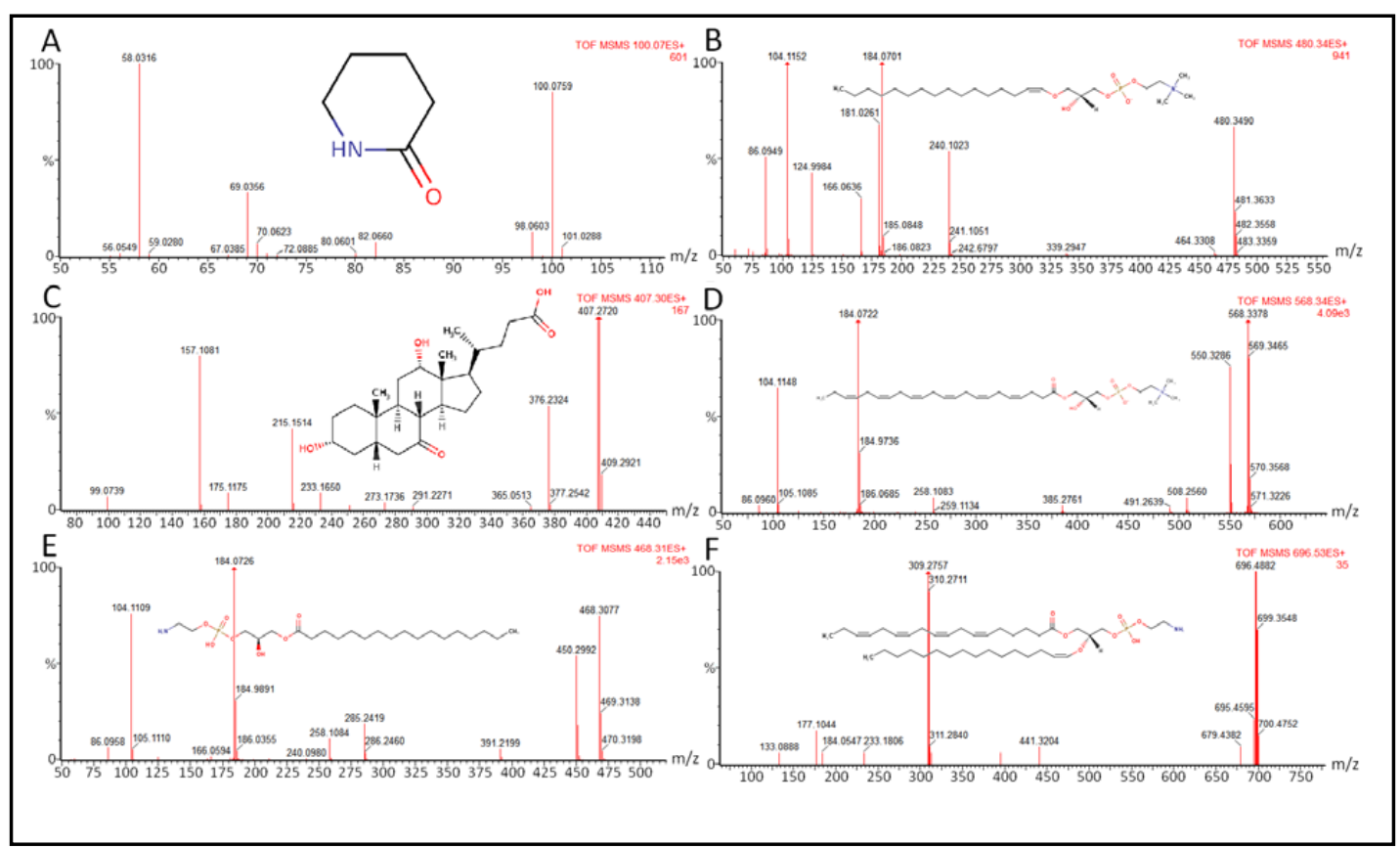

Fig. 5. Chemical structure and mass fragment information of 6 principal metabolites. (A) 2-Piperidinone, (B) 7-Ketodeoxycholicacid, (C) 1-Heptadecanoylglycerophosphoethanolamine, (D) LysoPC(P-16:0), (E) 2-Hexaprenyl-3-methyl-6-methoxy-1,4benzoquinone, and (F) LysoPC(22:6).

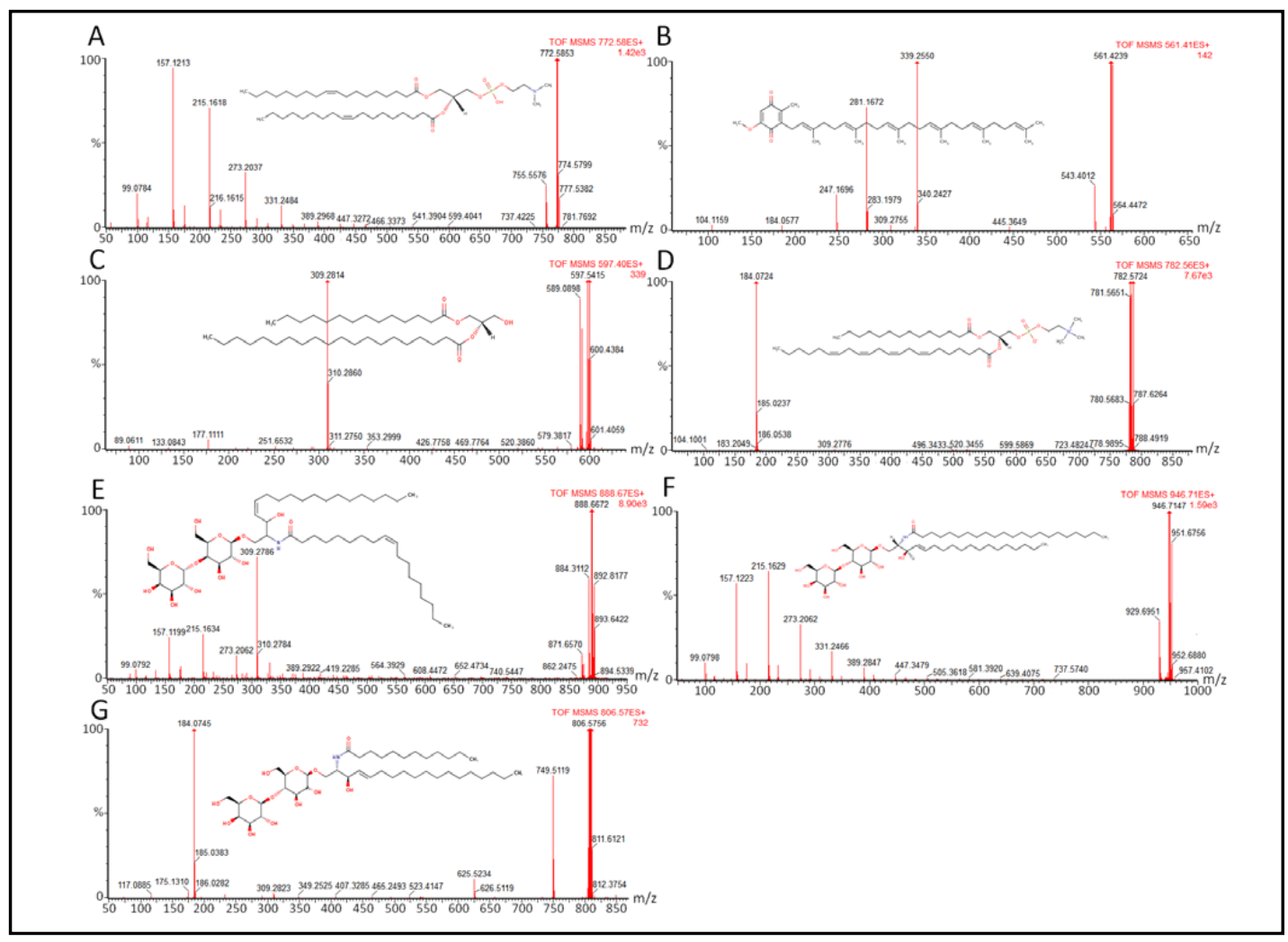

Fig. 6. Chemical structure and mass fragment information of 7 principal metabolites. (A) DG(14:0/20:0/0:0), (B) PE(18:4/P-16:0), (C) PE-NMe2(18:1/18:1), $\quad$ (D) PC(14:0/22:4), (E) 3-O-Sulfogalactosylceramide(d18:1/18:1), (F) Galabiosylceramide(d18:1/18:1), and (G) Lactosylceramide (d18:1/22:0). 
Table 5. Receiver operator characteristic curve analysis of 12 metabolites in positive ESI mode in the discovery study. AUC $=$ area under the curve, ${ }^{*}$ Sensitivity + specificty -1

\begin{tabular}{|c|c|c|c|c|c|}
\hline Biomarkers & AUC & $\begin{array}{c}\text { Best cutoff } \\
\text { (normalized } \\
\text { peak intensity) }\end{array}$ & Sensitivity, \% & $\%$ Specificity, \% & $\begin{array}{l}\text { Maximum of } \\
\text { Youden index* }\end{array}$ \\
\hline 2-Piperidinone & 0.793 & 126.17 & 73 & 83 & 0.57 \\
\hline Eicosadienoic acid & 0.768 & 339.05 & 63 & 93 & 0.57 \\
\hline 7-Ketodeoxycholic acid & 0.673 & 49.76 & 87 & 57 & 0.43 \\
\hline Varanic acid & 0.676 & 25.80 & 67 & 70 & 0.37 \\
\hline 1-Heptadecanoylglycerophosphoethanolamine & 0.856 & 17.45 & 97 & 60 & 0.57 \\
\hline LysoPC(15:0) & 0.737 & 60.71 & 77 & 70 & 0.47 \\
\hline LysoPE(0:0/20:0) & 0.763 & 172.80 & 70 & 77 & 0.47 \\
\hline $\begin{array}{l}\text { 2-Hexaprenyl-3-methyl-6-methoxy-1,4 } \\
\text { benzoquinone }\end{array}$ & 0.746 & 54.75 & 70 & 73 & 0.43 \\
\hline $\operatorname{DG}(14: 0 / 20: 0 / 0: 0)$ & 0.732 & 31.84 & 67 & 70 & 0.37 \\
\hline $\operatorname{PC}(14: 1 / 22: 2)$ & 0.822 & 92.13 & 90 & 57 & 0.47 \\
\hline Galabiosylceramide (d18:1/18:1) & 0.802 & 100.63 & 83 & 63 & 0.47 \\
\hline Lactosylceramide (d18:1/22:0) & 0.747 & 74.54 & 77 & 67 & 0.43 \\
\hline
\end{tabular}

Table 6. Multiple logistic regression models for the discovery and validation cohorts. $\mathrm{SE}=$ standard error * $\mathrm{R} 2=0.606+\mathrm{R} 2=0.799$

\begin{tabular}{|c|c|c|c|c|c|c|c|}
\hline \multirow{2}{*}{ Factor } & \multicolumn{4}{|c|}{ Discovery cohort* } & \multicolumn{3}{|c|}{ Validation cohort $\dagger$} \\
\hline & Estimate & SE & Wald $\chi 2$ & p value & Estimate & SE & Wald $\chi 2 \mathrm{p}$ value \\
\hline Age & 0.0324 & 0.0344 & 0.8857 & 0.3467 & 0.0423 & 0.0759 & 0.30990 .5778 \\
\hline 2-Piperidinone & -0.0119 & 0.0058 & 4.3084 & 0.0379 & -0.0352 & 0.0176 & 5.98020 .0460 \\
\hline 1-Heptadecanoylglycerophosphoethanolamine & 0.0955 & 0.0322 & 8.7769 & 0.0031 & 0.0992 & 0.0476 & 4.33580 .0373 \\
\hline
\end{tabular}

Table 7. ROC analysis in discovery and validation stage using a discovery model with 2 factors. ROC = receiver operator characteristic; $\mathrm{AUC}=$ area under the curve

\begin{tabular}{lccccc}
\hline Sample & AUC (95\%CI) & \multicolumn{1}{c}{ Best cutoff (P of logistic) } & Sensitivity, \% Specificity, \% Maximum of Youden index* \\
\hline Discovery $0.894(0.814-0.971)$ & 0.284 & 96.7 & 66.7 & 0.633 \\
Validation $0.956(0.891-1.000)$ & 0.336 & 93.3 & 80.0 & 0.733 \\
\hline
\end{tabular}

(AUC > 0.6). In particular, 2-piperidinone and 1-heptadecanoylglycerophosphoethanolamine showed excellent predictability of disease status (AUC > 0.75). The sensitivity, specificity, and best cutoff according to the maximum value of the Youden index were also determined (Table 5).

\section{Multiple logistic regression analysis}

To identify the most representative biomarkers for distinguishing OC patients from healthy people, a multiple logistic regression model was established by stepwise selection. The model was first established with 12 metabolites in the discovery samples after controlling for age. Two metabolites, i.e., 2-piperidinone $(\beta=-0.0119, \mathrm{P}<0.05)$ and 1-heptadecanoylglycerophosphoethanolamine $(\beta=0.0955, P<0.05)$, were also included in the model generated with the independent validation samples (2-piperidinone, $\beta=-0.0352$, $\mathrm{P}<0.05$ and 1-heptadecanoylglycerophosphoethanolamine, $\beta=0.0992, \mathrm{P}<0.05$ ) (Table 6). We calculated the sensitivity, specificity, and AUC of the regression model and the results showed that the model fit well with the discovery sample (AUC $=0.894$; sensitivity $=96.7 \%$; specificity $=66.7 \%$ ) and validation sample $($ AUC $=0.956$; sensitivity $=93.3 \%$; specificity $=$ 80.0\%) (Table 7 and Fig. 7). 
Fig. 7. ROC analysis in discovery and validation stages using a discovery model for the combination of two biomarkers. Blue line and red line were finished in the discovery and validation samples, and respectively.

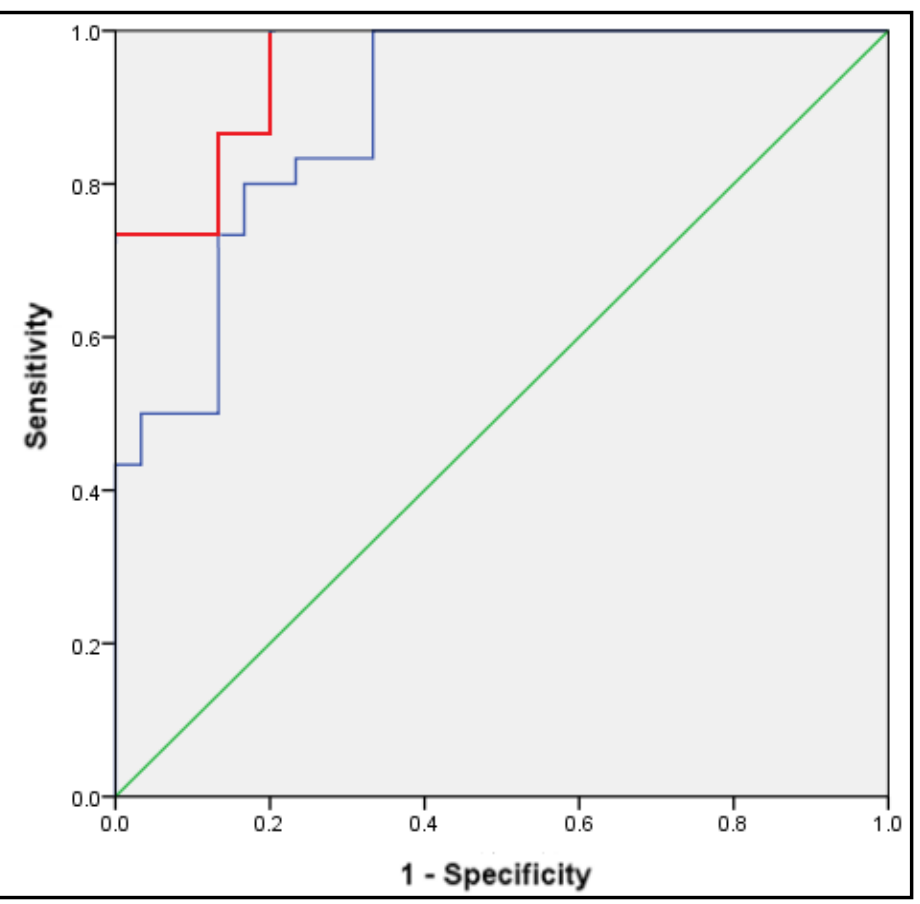

\section{Discussion}

In this study, we carried out a metabolomics analysis of serum samples from OC patients to identify potential biomarkers and determined that 18 metabolites best characterized disease status, of which 12 were validated in an independent case-control study. Multiple logistic regression analysis of the 12 metabolites showed that two, 2-piperidinone and 1-heptadecanoylglycerophosphoethanolamine, were significant predictors of OC.

Metabolic pathway analysis of the 12 identified metabolites showed that they were closely associated with OC. The 2-piperidinone is a derivative of piperidine, which is an organic compound that affects the activity and expression of cytochrome P450 (CYP)2E1, and thus indirectly influences oxidative stress and metabolic activation of low-molecular weight toxins [18]. CYP2E1 is associated with the metabolism of a variety of compounds including 1, 3-butadiene, nitrosamines, benzene, and other carcinogens, and free radicals [19]. CYP2E1 is implicated in human malignancies including gastrointestinal and respiratory tumors [20,21], and CYP2E1 gene polymorphisms are associated with the occurrence of breast and cervical cancers in females [22,23]. Another study showed that 2-piperidone is a specific marker of OC [24], which is in accordance with our findings.

Eicosadienoic acid is an omega- 6 fatty acid. The n-6 PUFA is a specific substrate for 5-lipoxygenase (5-LOX) and cyclooxygenase (COX)-2. Studies have shown that n-6 PUFA interacts with 5-LOX and COX-2 on the cell membrane to generate arachidonic acid compounds such as prostaglandin E2 and 12-hydroxy-carbon tetracon acid, which are known to promote cancer. Elevated levels of 5-LOX and COX-2 in breast cancer cells enhance the proliferation, invasion, and migration of cancer cells by activating $\mathrm{G}$ protein-coupled receptors (GPCRs), promoting tumor angiogenesis, and inhibiting apoptosis and the immune system [25]. Additionally, n- 6 fatty acid-derived metabolites promote angiogenesis, a necessary step for tumor growth and metastasis, by inducing the expression of growth factors [26]; n- 6 fatty acids have also been implicated in the progression of breast [27] and prostate [28] cancers.

The compound 1-heptadecanoylglycerophosphoethanolamine, lysoPC (15:0), LysoPE (0:0/20:0), and PC (14:1/22:2) are phosphate esters like lysophosphatidic acid (LPA), a natural phospholipid that promotes the invasion, metastasis, and proliferation of cancer 


\section{Cellular Physiology Cell Physiol Biochem 2018;51:1134-1148

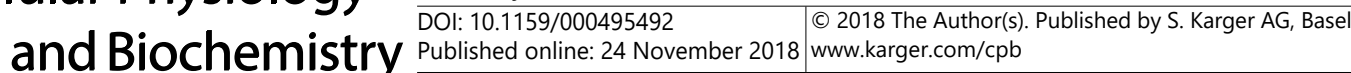 \\ Yang et al.: Metabolic Profiling of Serum in Ovarian Cancer}

cells. A previous study showed that LPA can induce OC cell proliferation and adhesion junction breakdown via activation of Src family kinases [29]. LPA stimulates SKOV3 OC cell proliferation and inhibits their apoptosis through activation of mitogen-activated protein kinase signaling [30]. Another study demonstrated that LPA induces the migration, invasion, and proliferation of OC cells through regulation of vascular endothelial growth factor, urokinase plasminogen activator, matrix metalloproteinases, interleukin-8, COX-2, interleukin-6, CXC motif chemokine ligand 12/CXC receptor 4, cyclin D1, Hippo-Yap, and growth-regulated oncogene $\alpha$ [31].

Lactosylceramide and galabiosylceramide are ceramides, which are potent suppressors of neoplasms that promote apoptosis, autophagy, and cell cycle arrest [32]. One study reported that ceramide glycosylation affected cancer development and progression by regulating gene expression, restoring p53 expression via RNA splicing, and downregulating multidrug-resistant 1 via cSrc/ $\beta$-catenin signaling [33]. It has also been suggested that fatty acid chain length, subcellular localization, and/or direct downstream targeting of de novogenerated ceramides have distinct and opposite effects in tumor promotion/suppression [34].

Ketodeoxycholic acid and varanic acid are bile acids whose accumulation in blood and tissues is controlled by various mechanisms owning to their toxicity [35, 36]. Bile acids such as chenodeoxycholic acid (CDCA) and DCA are cytotoxic to OC cells and induce their apoptosis [37]. Most bile acids are cytotoxic to a broad range of tumor cells and are highly selective for HepG2 cells, with CDCA exhibiting especially high selectivity for these cells [38]. A reduction in CDCA concentrations was found to accelerate human endometrial cancer cell growth by inducing an increase in cyclin D1 protein and mRNA expression via activation of GPCR-dependent pathways [39]. The close relationship between bile acids and colorectal cancer [40] and gastric cancer [41] in females provide support for bile acid levels as a predictor of OC.

The 2-hexaprenyl-3-methyl-6-methoxy-1, 4 benzoquinone is a polyprenyl benzoquinone that is involved in the ubiquinone (CoQ) biosynthesis pathway. CoQ10 influences the expression of genes involved in cell signaling, metabolism, and trafficking, which may in turn indirectly promote cancer onset [42]. Epidemiological studies have shown that low plasma concentrations of CoQ10 may be caused by an inadequate dietary intake or reduced endogenous biosynthesis resulting from increased utilization of reactive oxygen species induced by oxidative stress [43]. DG (14:0/20:0/0:0) is a diglyceride or a diacylglycerol (DAG) composed of two fatty acid chains covalently bound to a glycerol molecule via ester linkages. Diglycerides play an important role in signal transduction and lipid metabolism in mammalian cells [44]. Diacylglycerol kinase (DGK) $\alpha$ is one of 10 DGK family members that convert diglycerides into phosphatidic acid, which along with DAG is a lipid second messenger in the plasma membrane. DGK $\alpha$ inhibition can promote $\mathrm{T}$ cell activation and boost cancer immunotherapy by preventing angiogenesis and causing damage to cancer cells [45].

\section{Conclusion}

In summary, we identified 12 biomarkers that may be associated with abnormal fatty acid $\beta$-oxidation and phospholipid and bile acid metabolism in OC using a UPLC/QTOF MS-based metabolomics strategy and multivariate data analysis. Two of the identified metabolites (2-piperidinone and 1-heptadecanoylglycerophosphoethanolamine) were validated in a multiple logistic regression model. These findings provide novel insight into the pathogenesis of $\mathrm{OC}$, and may be applicable to its clinical diagnosis and treatment. 


\section{Cellular Physiology Cell Physiol Biochem 2018;51:1134-1148 and Biochemistry Published $\begin{aligned} & \text { DOI } 10.1159 / 000495492 \\ & \text { (c) } 2018 \text { The Author(s). Published by S. Karger AG, Basel } \\ & \text { www.karger.com/cpb }\end{aligned}$}

Yang et al.: Metabolic Profiling of Serum in Ovarian Cancer

\section{Acknowledgements}

This work was supported by grants from the Science Foundation of Heilongjiang Province (LC2016032), and Youth Foundation in School of Public Health of Harbin Medical University (201511).

\section{Disclosure Statement}

The authors declare that they have no competing interests.

\section{References}

1 Rauh-Hain JA, Krivak TC, Del Carmen MG, Olawaiye AB: Ovarian cancer screening and early detection in the general population. Rev Obstet Gynecol 2011;4:15-21.

Auersperg N: The origin of ovarian cancers--hypotheses and controversies. Front Biosci 2013;S5:709-719. Ben Sellem D, Elbayed K, Neuville A, Moussallieh FM, Lang-Averous G, Piotto M, Bellocq JP, Namer IJ: Metabolomic Characterization of Ovarian Epithelial Carcinomas by HRMAS-NMR Spectroscopy. J Oncol 2011; 2011:174019.

4 Jacobs I J, Menon U: Progress and challenges in screening for early detection of ovarian cancer. Mol Cell Proteomics 2004;3:355-366.

5 Nicholson JK, Lindon JC and Holmes E: 'Metabonomics': understanding the metabolic responses of living systems to pathophysiological stimuli via multivariate statistical analysis of biological NMR spectroscopic data. Xenobiotica 1999;29:1181-1189.

6 Chen G, Ye G, Zhang X, Liu X, Tu Y, Ye Z, Liu J, Guo Q, Wang Z, Wang L, Dong S, Fan Y: Metabolomics Reveals Protection of Resveratrol in Diet-Induced Metabolic Risk Factors in Abdominal Muscle. Cell Physiol Biochem 2018;45:1136-1148.

7 Ren Y, Tang Q, Liu W, Tang Y, Zhu R, Li B: Serum Biomarker Identification by Mass Spectrometry in Acute Aortic Dissection. Cell Physiol Biochem 2017;44:2147-2157.

8 Johnson CH, Patterson AD, Idle JR, Gonzalez FJ: Xenobiotic metabolomics: major impact on the metabolome. Annu Rev Pharmacol Toxicol 2012;52:37-56.

-9 Chen J, Zhang X, Cao R, Lu X, Zhao S, Fekete A, Huang Q Schmitt-Kopplin P, Wang Y, Xu Z, Wan X, Wu X, Zhao N, Xu C, Xu G: Serum 27-nor-5ß-Cholestane-3, 7,12, 24, 25 Pentol Glucuronide Discovered by Metabolomics as Potential Diagnostic Biomarker for Epithelium Ovarian Cancer. J Proteome Res 2011;10:2625-2632.

10 Garcia E, Andrews C, Hua J, Kim HL, Sukumaran DK, Szyperski T, Odunsi K: Diagnosis of early stage ovarian cancer by $1 \mathrm{H}$ NMR metabonomics of serum explored by use of a microflow NMR probe. J Proteome Res 2011;10:1765-1771.

11 Yin R, Yang T, Su H, Ying L, Liu L, Sun C: Saturated fatty acids as possible important metabolites for epithelial ovarian cancer based on the free and esterified fatty acid profiles determined by GC-MS analysis. Cancer Biomark 2016, 17:259-269.

-12 O'Connor D, Mortishire-Smith R: High-throughput bioanalysis with simultaneous acquisition of metabolic route data using ultra performance liquid chromatography coupled with time-of-flight mass spectrometry. Anal Bioanal Chem 2006;385:114-121.

13 Wang M, Yang X, Wang F, Li R, Ning H, Na L, Huang Y, Song Y, Liu L, Pan H, Zhang Q Fan L, Li Y, Sun C: Calcium-deficiency assessment and biomarker identification by an integrated urinary metabonomics analysis. BMC Med 2013;11:86.

14 Teneriello MG, Park RC: Early detection of ovarian cancer. CA Cancer J Clin 1995;45:71-87.

15 Wang C, Feng R, Sun D, Li Y, Bi X, Sun C: Metabolic profiling of urine in young obese men using ultra performance liquid chromatography and Q-TOF mass spectrometry (UPLC/Q-TOF MS). J Chromatogr B Analyt Technol Biomed Life Sci 2011;879:2871-2876.

16 Wang C, Feng R, Li Y, Zhang Y, Kang Z, Zhang W, Sun DJ: The metabolomic profiling of serum in rats exposed to arsenic using UPLC/Q-TOF MS. Toxicol Lett 2014; 229:474-481. 


\section{Cellular Physiology Cell Physiol Biochem 2018;51:1134-1148 and Biochemistry \begin{tabular}{l|l} 
DOI: 10.1159/000495492 2018 The Author(s). Published by S. Karger AG, Basel \\
Publishect
\end{tabular}

17 Eriksson L, Johansson E, Kettaneh-Wold N, Trygg J, Wikstr C, Wold S: Multi-and megavariate data analysis part I: Basic principles and applications. Second revised and enlarged edition 2006;45:362-362.

$>18$ Cheng J, Chen C, Kristopher KW, Manna SK, Scerba M, Friedman FK, Luecke H, Idle JR, Gonzalez FJ: Identification of 2-Piperidone as a Biomarker of CYP2E1 Activity Through Metabolomic Phenotyping. Toxicol Sci 2013;135:37-47.

19 Rannug A, Alexandrie AK, Persson I, Ingelman-Sundberg M: Genetic polymorphism of cytochromes P450 1A1, 2D6 and 2E1: regulation and toxicological significance. J Occup Environ Med 1995;37:25-36.

-20 Zienolddiny S, Campa D, Lind H, Ryberg D, Skaug V, Stangeland LB, Canzian F, Haugen A.: A comprehensive analysis of phase I and phase II metabolism gene polymorphisms and risk of non-small cell lung cancer in smokers. Carcinogenesis 2008; 29:1164-1169.

-21 Sugimura T, Kumimoto H, Tohnai I, Fukui T, Matsuo K, Tsurusako S, Mitsudo K, Ueda M, Tajima K, Ishizaki K.: Gene-environment interaction involved in oral carcinogenesis: molecular epidemiological study for metabolic and DNA repair gene polymorphisms. J Oral Pathol Med 2006;35:11-18.

22 Ferreira PM, Catarino R, Pereira D, Matos A, Pinto D, Coelho A, Lopes C, Medeiros R: Cervical cancer and CYP2E1, polymorphisms: implications for molecular epidemiology. Eur J Clin Pharmacol. 2006; 62:15-21.

23 Wu SH, Tsai SM, Hou MF, Lin HS, Hou LA, Ma H, Lin JT, Yeh FL, Tsai LY: Interaction of genetic polymorphisms in cytochrome P450 2E1 and glutathione S-transferase M1 to breast cancer in Taiwanese woman without smoking and drinking habits. Breast Cancer Res Treat 2006;100:93-98.

24 Zhang T, Wu X, Yin M, Fan L, Zhang H, Zhao F, Zhang W, Ke C, Zhang G, Hou Y, Zhou X, Lou G, Li K: Discrimination between malignant and benign ovarian tumors by plasma metabolomic profiling using ultra performance liquid chromatography/mass spectrometry. Clin Chim Acta 2012;413:861-868.

25 Singh B, Berry JA, Shoher A, Ramakrishnan V, Lucci A: Cox-2 overexpression increases motility and invasion of breast cancer cells. Ann Surg Oncol 2004; 11:S51-S51.

-26 Kang JX, Liu A. The role of the tissue omega-6/omega-3 fatty acid ratio in regulating tumor angiogenesis. Cancer Metastasis Rev 2013;32:201-210.

-27 de Lorgeril M, Salen P. New insights into the health effects of dietary saturated and omega- 6 and omega-3 polyunsaturated fatty acids. BMC Med 2012;10:50.

28 Apte SA, Cavazos DA, Whelan KA, Degraffenried LA: A low dietary ratio of omega- 6 to omega-3 Fatty acids may delay progression of prostate cancer. Nutr Cancer 2013; 65:556-562.

29 Purow B: Molecular Pathways: Targeting Diacylglycerol Kinase Alpha in Cancer. Clin Cancer Res 2015; 21:5008-5012.

30 Huang RY, Wen CC, Liao CK, Wang SH, Chou LY, Wu JC: Lysophosphatidic acid modulates the association of PTP1B with N-cadherin/catenin complex in SKOV3 ovarian cancer cells. Cell Biol Int 2012;36:833-841.

-31 Sun H, Ren J, Zhu Q Kong FZ, Wu L, Pan BR: Effects of lysophosphatidic acid on human colon cancer cells and its mechanisms of action. World Journal of Gastroenterology 2009; 15:4547-4555.

-32 Morad SAF, Cabot MC: Ceramide-orchestrated signalling in cancer cells. Nat Rev Cancer 2013;13:51-65.

-33 Liu YY, Hill RA, Li YT: Ceramide glycosylation catalyzed by glucosylceramide synthase and cancer drug resistance. Adv Cancer Res 2013;117:59-89.

-34 Ponnusamy S, Meyers-Needham M, Senkal CE, Saddoughi SA, Sentelle D, Selvam SP, Salas A, Ogretmen B: Sphingolipids and cancer: ceramide and sphingosine-1-phosphate in the regulation of cell death and drug resistance. Future Oncol 2010;6:1603-1624.

-35 St-Pierre MV, Kullak-Ublick GA, Hagenbuch B, Meier PJ: Transport of bile acids in hepatic and non-hepatic tissues. J Exp Biol 2001; 204:1673-1686.

-36 Claudel T, Staels B, Kuipers F: The Farnesoid X receptor: a molecular link between bile acid and lipid and glucose metabolism. Arterioscler Thromb Vasc Biol 2005; 25:2020-2030.

37 Horowitz NS, Hua J, Powell MA, Gibb RK, Mutch DG, Herzog TJ: Novel cytotoxic agents from an unexpected source: bile acids and ovarian tumor apoptosis. Gynecol Oncol 2007;107:344-349.

-38 Gong Y, Zhang X, Zhang Y, Chu F, Li G, Zhang H, Li B, Wang P, Lei H: Bile acids, carriers of hepatoma-targeted drugs? Pharmazie 2016;71:139-145.

-39 Casaburi I, Avena P, Lanzino M, Sisci D, Giordano F, Maris P, Catalano S, Morelli C, Andò S: Chenodeoxycholic acid through a TGR5-dependent CREB signaling activation enhances cyclin D1 expression and promotes human endometrial cancer cell proliferation. Cell Cycle 2012;11:2699-2710.

40 Cross AJ, Moore SC, Boca S, Huang WY, Xiong X, Stolzenberg-Solomon R, Sinha R, Sampson JN: A prospective study of serum metabolites and colorectal cancer risk. Cancer 2015;120:3049-3057. 


\section{Cellular Physiology Cell Physiol Biochem 2018;51:1134-1148 and Biochemistry \begin{tabular}{c|c} 
DOl: 10.1159/000495492 \\
Published 2018 The Author(s). Published by S. Karger AG, Basel
\end{tabular}

Yang et al.: Metabolic Profiling of Serum in Ovarian Cancer

41 Chen MC, Chen YL, Wang TW, Hsu HP, Lai MD: Membrane bile acid receptor TGR5 predicts good prognosis in ampullary adenocarcinoma patients with hyperbilirubinemia. Oncol Rep 2016;36:1997-2008.

-42 Garrido-Maraver J, Cordero MD, Oropesa-Avila M, Vega AF, de la Mata M, Pavon AD, Alcocer-Gomez E, Calero CP, Paz MV, Alanis M, de Lavera I, Cotan D, Sanchez-Alcazar JA: Clinical applications of coenzyme Q10 Front Biosci 2014; 19:619-633.

43 Palan PR, Mikhail MS, Shaban DW, Romney SL: Plasma concentrations of coenzyme Q10 and tocopherols in cervical intraepithelial neoplasia and cervical cancer. Eur J Cancer Prev 2003;12:321-326.

44 Yang K, Jenkins CM, Dilthey B, Gross RW: Multidimensional Mass Spectrometry-based Shotgun Lipidomics Analysis of Vinyl Ether Diglycerides. Anal Bioanal Chem 2015;407:5199-5210.

45 Bhat K P, Aldape K: Not expecting the unexpected: diacylglycerol kinase alpha as a cancer target. Cancer Discovery 2013;3:726-727. 